\title{
Four-dimensional flow magnetic resonance imaging is useful for assessing hemolytic anemia following surgical repair of type A aortic dissection
}

Kenichiro Takahashi ${ }^{1}$, Tetsuro Sekine ${ }^{2}$, Jiro Kurita ${ }^{3}$, and Yosuke Ishii ${ }^{1}$

${ }^{1}$ Nippon Medical School

${ }^{2}$ Nippon medical school Musashi Kosugi hospital

${ }^{3}$ Affiliation not available

August 11, 2021

\begin{abstract}
In a case of mechanical hemolytic anemia following surgical repair of type A aortic dissection, four-dimensional flow magnetic resonance imaging revealed highly elevated turbulent kinetic energy in a stenotic lesion of a replaced ascending aorta. Our findings suggest that turbulent kinetic energy evaluation enables the detection of the origin of mechanical hemolysis.
\end{abstract}

Four-dimensional flow magnetic resonance imaging is useful for assessing hemolytic anemia following surgical repair of type A aortic dissection

Kenichiro Takahashi, $\mathrm{MD}^{\mathrm{a}}$

Tetsuro Sekine, $\mathrm{MD}, \mathrm{PhD}^{\mathrm{b}}$

Jiro Kurita, MD, $\mathrm{PhD}^{\mathrm{a}}$

Yosuke Ishii, $\mathrm{MD}, \mathrm{PhD}^{\mathrm{a}}$

${ }^{\text {a }}$ Cardiovascular Surgery, Nippon Medical School, Tokyo, Japan

b Radiology, Nippon Medical School Musashi Kosugi Hospital, Kanagawa, Japan

Running title: $4 \mathrm{D}$ flow for hemolysis

Corresponding:

Kenichiro Takahashi

Cardiovascular Surgery, Nippon Medical School

1-1-5 Sendagi, Bunkyo-ku, Tokyo 113-8603, Japan

Tel: $+81-3-3822-2131$

E-mail: takahashi-ken@nms.ac.jp

Conflict of Interest : None

Funding: This work was supported by JSPS KAKENHI (Grant Number 17K18160, 19K17151 and 19K08186), research grants from Fukuda Foundation for Medical Technology and Terumo foundation for life sciences and arts. 
IRB approval and clinical trial registration are not applicable for this study.

\begin{abstract}
In a case of mechanical hemolytic anemia following surgical repair of type A aortic dissection, fourdimensional flow magnetic resonance imaging revealed highly elevated turbulent kinetic energy in a stenotic lesion of a replaced ascending aorta. Our findings suggest that turbulent kinetic energy evaluation enables the detection of the origin of mechanical hemolysis.
\end{abstract}

Four-dimensional flow magnetic resonance imaging (4D flow MRI) has been developed to assess flow derangement by visualizing pathlines. It can directly estimate turbulent kinetic energy (TKE) which is associated with pressure loss caused by stenotic lesion. ${ }^{1}$ We hereby report a case of hemolytic anemia following aortic surgery assessed by 4D flow MRI. Written informed consent was provided by the patient for the publication.

A 55-year-old woman with acute type A aortic dissection underwent ascending aortic replacement. The patient developed hemolytic anemia 2 weeks post-operatively. Computed tomographic angiography suggested two possible stenotic lesions, one was a kinked graft (Figure 1A) and the other was stenosed proximal anastomosis (Figure 1B).

The patient underwent 4D flow MRI examination which revealed jet flow (Figure 1C) and highly elevated TKE at the proximal anastomosis (Figure 1D) while no accelerated flow or TKE elevation was observed at the kinking point. The patient underwent re-operation to repair the proximal anastomosis, which was stenosed owing to inversion of the inner felt strip (Figure 2). Post-operative 4D flow MRI revealed smooth laminar flow and TKE reduction (Figure 3). The hemolytic anemia resolved immediately.

In conclusion, 4D flow MRI is useful for assessing hemolysis after aortic surgery. Our findings suggest that TKE evaluation enables the detection of the origin of mechanical hemolysis.

\title{
Legends
}

Figure 1: Computed tomographic angiography showed a kinked graft $(A)$ and stenosed proximal anastomosis $(B$, arrow ). 4D flow MRI revealed jet flow $(C$, arrow $)$ and highly elevated TKE $(D$, arrow $)$ at the proximal anastomosis, suggesting the severely stenosed proximal anastomosis as the cause of the mechanical hemolysis.

Figure 2: During re-operation, the proximal anastomosis was found to be stenosed owing to inversion of the inner felt strip (arrow).

Figure 3: Post-operative 4D flow MRI showed smooth laminar flow $(A)$ and TKE reduction

$(B)$ in the replaced ascending aorta.

\section{Reference}

1. Iwata K, Matsuda J, Imori Y, Sekine T, Takano H. Four-dimensional flow magnetic resonance imaging reveals the reduction in turbulent kinetic energy after percutaneous transluminal septal myocardial ablation in hypertrophic obstructive cardiomyopathy. Eur Heart J.2020;41(14):1454. 


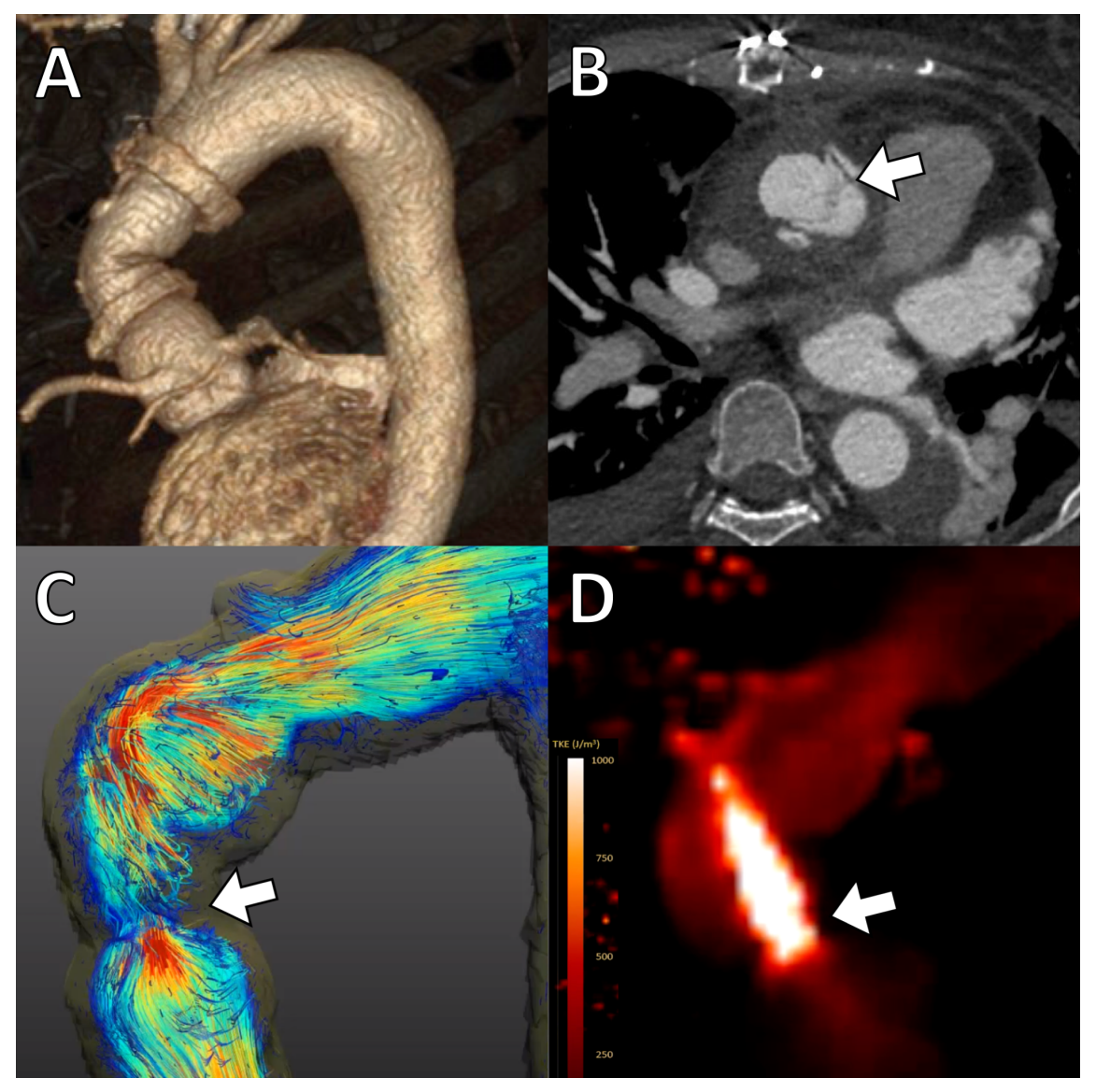



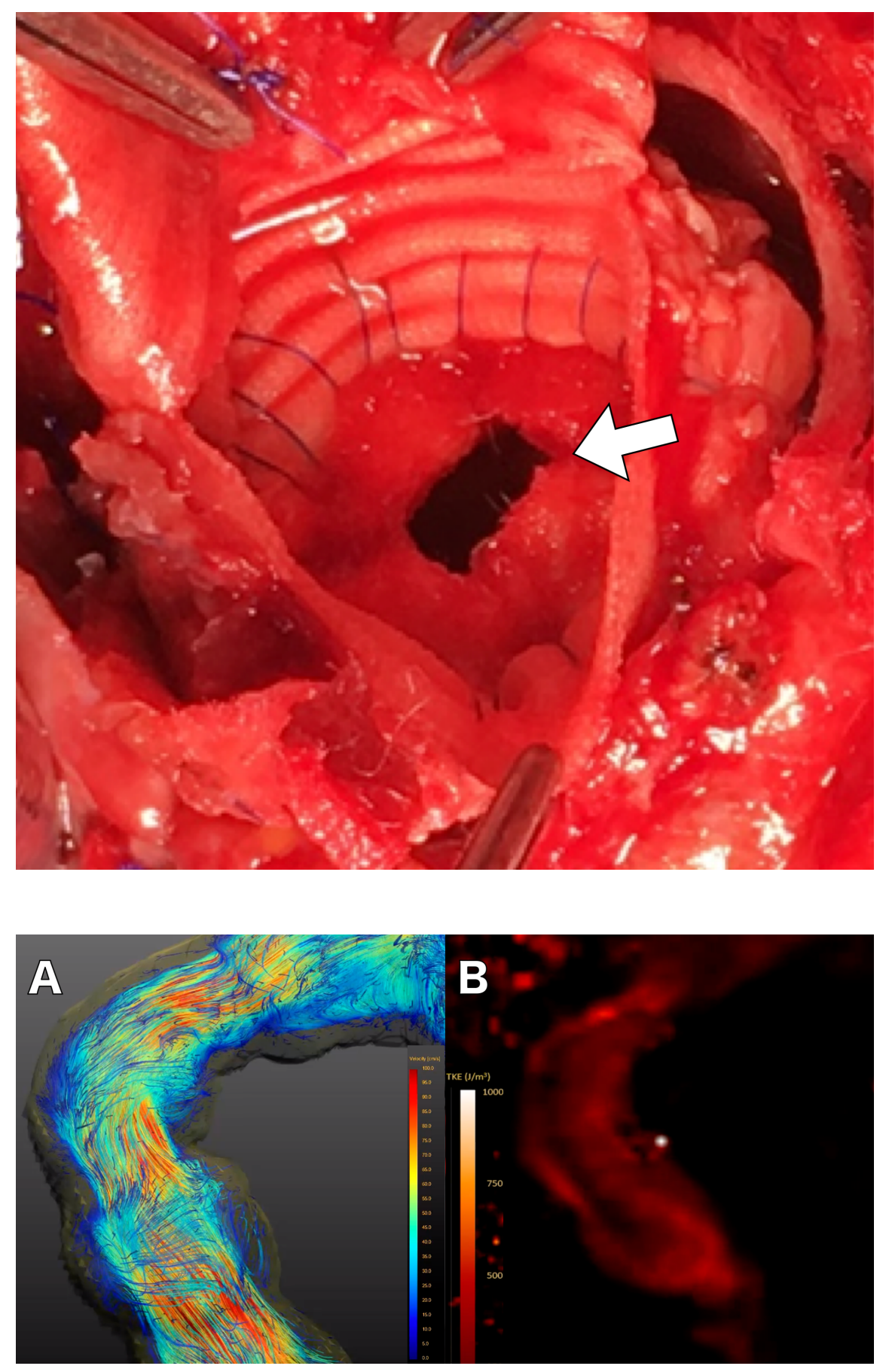